\title{
CARACTERIZACIÓN DE LA GERENCIA EN LA DESERCIÓN ESTUDIANTIL EN INSTITUCIONES DE EDUCACIÓN SUPERIOR DEL ATLÁNTICO, COLOMBIA \\ Rocío Gutiérrez Echeverría $^{1^{*}}$ \\ ${ }^{1}$ Universidad Simón Bolívar, Barranquilla, Colombia
}

*Autor de correspondencia: regutierrez@unisimonbolivar.edu.co

Recibido Septiembre 2020; Aceptado Diciembre 2020

\begin{abstract}
Resumen - El estudio tiene como propósito caracterizar la gerencia en la deserción estudiantil en instituciones de educación superior del sector privado de la ciudad de Barranquilla, Departamento del Atlántico - Colombia. Se estructuró con base en categorías y subcategorías que describen elementos importantes que abordan la temática gerencial y el fenómeno de la deserción estudiantil. Esta investigación se realizó bajo el paradigma epistemológico cualitativo, específicamente con el método fenomenológico, se aplicó un guion de entrevista con ciertos requisitos para la selección de los dicientes entrevistados, los cuales fueron tres sujetos con cargo de coordinador académico en las sedes de la Universidad Simón Bolívar de Barranquilla. En cuanto a la técnica de análisis de datos se utilizó la categorización axial que permite la codificación y categorización selecta mediante los procesos de integración y refinamiento de las categorías para explicar lo hallazgos del fenómeno, por consiguiente se teorizan los aportes y recomendaciones. Los resultados evidencian las deficientes competencias en habilidades gerenciales ante el problema de la deserción estudiantil; siendo los factores del mismo determinados por aspectos individuales, socioeconómicos, académicos e institucionales; y finalmente tipificarla por cohorte, período y ausencia intersemestral; por lo que se concluye que se concluye que los hallazgos se ubican dentro de los postulados teóricos y referenciales, por tratarse de directrices emanadas por el Ministerio de Educación Nacional de Colombia.
\end{abstract}

Palabras clave: Gerencia, deserción estudiantil, instituciones de educación superior, habilidades gerenciales, factores.

\section{CHARACTERIZATION OF MANAGEMENT IN STUDENT DESERTION IN HIGHER EDUCATION INSTITUTIONS OF ATLANTIC, COLOMBIA}

\begin{abstract}
The purpose of the study is to characterize the management of student desertion in higher education institutions of the private sector in the city of Barranquilla, Department of Atlántico - Colombia. It was structured based on categories and subcategories that describe important elements that address the management issue and the phenomenon of student desertion. This research was carried out under the qualitative epistemological paradigm, specifically with the phenomenological method. An interview script was applied with certain requirements for the selection of the interviewed students, who were three subjects in charge of the academic coordinator at the Simón Bolívar University in Barranquilla. As for the technique of data analysis, axial categorization was used, which allows the selected codification and categorization through the processes of integration and refinement of the categories to explain the findings of the phenomenon, therefore the contributions and recommendations are theorized. The results show the deficient competences in managerial skills in front of the problem of student
\end{abstract}


desertion; being the factors of it determined by individual, socioeconomic, academic and institutional aspects; and finally, typifying it by cohort, period and intersemestral absence; for what it is concluded that the findings are located within the theoretical and referential postulates, because they are guidelines emanated by the Ministry of National Education of Colombia.

Keywords: Management, student dropouts, higher education institutions, management skills, factors.

\section{Introducción}

En América Latina, cada día la deserción universitaria, es más prominente debido a los múltiples factores que afectan a los estudiantes de primeros semestres, el inicio de la adaptación a la vida universitaria, en el campus de pregrado proporcionan un escenario complejo, donde la vocación profesional, la perspectiva de la misma, la formación primaria y los factores socio económicos, entre otros, hacen que el estudiante se sienta rezagado, deseando abortar en el sistema educativo en sus primeros años, convirtiendo la oportunidad de su formación profesional en índices de deserción institucionales, nacionales e internacionales.

Ésta problemática que experimenta el sistema educativo, por consiguiente, los jóvenes de muy temprana edad los cuales desean plasmar sus metas profesionales, enfocada en la toma de decisiones propias se sienten persuadidos por sus padres u otros actores cercanos en su familia, haciendo del joven de respuestas impropias de sus responsabilidades adquiridas: según culturas se dan unas u otras dominancias en las actitudes y en los comportamientos.

Para el Ministerio de Educación Nacional Colombiano (2014), el concepto de desertor, corresponde al estudiante que no presenta matrícula durante dos períodos consecutivos o más al momento del estudio. Asimismo, el Sistema para la Prevención de la Deserción en Educación Superior (SPADIES), permite clasificar tres diferentes desertores: en primer lugar, el desertor de programa, como aquel estudiante que no se matricula en la misma carrera, durante el periodo antes señalado como mínimo, aun cuando se mantenga en la misma institución educativa.

La segunda clasificación, es el desertor de la Institución de Educación Superior (IES), referido a aquel estudiante que no se matricula en una institución educativa durante dos períodos consecutivos o más al momento del estudio. En tal sentido, el estudiante presenta matrícula en otra institución educativa diferente a la que lo registró como primíparo; y la tercera clasificación, corresponde al desertor de sistema, aquel que no se matricula en ninguna institución educativa, durante dos períodos consecutivos o más al momento del estudio.

En consecuencia, el retraso en los estudios y la deserción universitaria, son problemas de un alto índice nacional e internacional. En Europa, es notable la deserción universitaria en cada ciclo inicial de matrículas, los bajos rendimientos académicos, son cada vez más prominentes, sin embargo, las últimas tendencias marcan los intercambios estudiantiles, pasantías, y estudiantes, favorecidos con becas para estudiar en el exterior, esto reflejado en un bajo porcentaje, pero ha sido frecuente el acceso.

Por ende, el mayor crecimiento de los índices de deserción universitaria, se inició hace poco más de cuatro décadas, se fue agravando en los noventa. La tasa de abandono para la costa Caribe IPSA SCIENTIA - Vol. 5 No $1-2020$ 
colombiana fue de 15,10\%, y a nivel nacional fue de 12, $12 \%$ en el 2014, sin embargo, para este último año comprendido entre el segundo periodo del 2014 y primer semestre del año 2015, la deserción tuvo un incremento del 18\%, las cifras en los países desarrollados no están lejos de la realidad del sistema actual.

En continuidad con lo anterior, en España oscilan entre 30\% y 50\%, son muy similares a las de otros países como Francia, Austria y Estados Unidos de Norteamérica. Sin embargo, en Alemania, son algo más bajas (20-25\%), en Suiza se presenta una deserción del (7\%-30\%), Finlandia con un (10\%) y Países Bajos (20\%-30\%). No es casualidad que las tasas de abandono sean inferiores en los países con sistemas más selectivos, presentando resultados superiores en aquéllos que tienen un sistema más abierto (Gutiérrez et al., 2018).

Por lo tanto, el propósito del Plan Nacional de Desarrollo 2014 - 2018: “Todos por un nuevo país”, es construir una Colombia en paz, equitativa y educada; es decir, una sociedad que cuenta con una fuerza laboral calificada, sin grandes diferencias de ingresos, con ciudadanos que resuelven sus conflictos sin recurrir a la violencia, la paz favorece la equidad y la educación, la equidad propicia la paz y la educación, y la educación genera condiciones de paz y equidad (Ley 1753, 2015).

En este contexto, Rodríguez et al. (2017), indica que la educación, incide en el crecimiento y desarrollo de un país, donde sus ingresos apuntan al aumento de la demanda por educación, impactando positivamente la productividad y el impulso de una educación pertinente para acelerar el crecimiento, bien sea la educación formal general, el entrenamiento técnico o la educación informal relacionada con trabajos específicos (Agudelo, 2008).

Del mismo modo, Malagón \& Reverón (2001), la cobertura universitaria en el marco de las universidades, es de gran preocupación e importancia ya que el orden y cumplimiento de las normas y políticas institucionales, deben verse reflejada en el cumplimiento de las mismas, esto mediante del sistema histórico y el seguimiento de matrícula de la comunidad educativa, no obstante, son detectados signos de injusticia e inequidad, donde todo ellos debe apuntar a la contribución para que dicho orden cambie o se transforme (Franco-Gallego, 2019).

Como lo describen los autores, la implementación al cambio, no es viable, si no se comprende la manera como se estructura o compone dicho orden, cuando el campo del sistema educativo, es complejo, donde es de vital importancia la comprensión de las condiciones históricas, sociales políticas que producen y profundizan las desigualdades en la sociedad.

En este aspecto, el compromiso de las instituciones educativas, debe ser plantear y ejecutar estrategias para llevar a cabo la culminación de los estudios en los años programados, esto mediante programas institucionales internos, que fortalezcan el aprendizaje adquirido de acuerdo a su formación profesional o plan de estudios, respaldado por las políticas institucionales, llevando a cabo el cumplimiento misional, de esta manera, uno de los factores de deserción que se puntualiza en la academia, es el bajo rendimiento académico, el cual sería fortalecido mediante los distintos programas estratégicos, al igual se debe plantear estrategias que contrarresten o disminuyan las causas socio económicas (Fong-Silva et al., 2018).. 
Sobre la base de estos planteamientos, el presente artículo persigue caracterizar la gerencia en la deserción estudiantil en instituciones de educación superior del sector privado de la ciudad de Barranquilla, Departamento del Atlántico - Colombia.

En relación, al rezago el cual es el tiempo en que dura un estudiante en culminar su carrera, estos jóvenes presentan una particularidad que comparten con los estudiantes permanentes, los cuales son influenciados por subgrupos que desarrollan otras actividades, como espacios deportivos, grupos religiosos, político, entre otros, llevándolos a perder la motivación por la meta trazada de su formación profesional, dándole prioridad a otras actividades, asiéndoles desistir incluso de aplazar semestres o reincidir en el mismo, esto trae como consecuencia un impacto negativo en su círculo social e incluso para el mismo.

Todo ello sin tener en cuenta las vulnerabilidades, inconvenientes, crisis u obstáculos que se hayan presentado en el tiempo asignado para la culminación de los estudios, la satisfacción, de haber culminado en el tiempo, con su misma corte y el acoplamiento obtenido mediante la disciplina de estudio, son factores que influyen positivamente en la seguridad y proyección del profesional, desarrollo de habilidades del pensamiento y experiencias de aprendizajes.

En continuidad con los planteamientos mencionados, Clifton, Perry, Adams \& Roberts (2008), citado por Kim \& Sax (2017) desarrollan una forma de pensar más liberal y crítica, y adquisición de un conjunto de destrezas y habilidades que lo preparan para afrontar la productividad, desarrollo y solución de problemas, entre otros. Por consiguiente, son los retos personales los que impulsan a los estudiantes a desenvolverse efectivamente en un competitivo ambiente laboral. No obstante, la relación entre modelos pedagógicos débiles y hábitos individuales deficientes hará que el estudiante abandone el sistema educativo.

De acuerdo a lo anterior, cabe resaltar que las instituciones u organizaciones universitarias, son estructuradas como una red compleja de procesos, donde se presentan eventos interrelacionados con la planeación organizacional inteligente, dirección y control de los recursos humanos, también integrado con el sistema abierto socio-técnico, donde se relacionan los aspectos tecnológicos como las herramientas de software u equipos de hardware inter relacionados con los recursos humanos, que dan respuesta a toma decisiones transformadoras en objetivos organizacionales o mecanismos propios misionales de las instituciones, todo ello interconectado de manera compleja, en constante cambio y bajo la presión de diferentes escenarios del entorno.

En este sentido, se puede ver claramente que los sistemas de gestión de las instituciones u organizaciones educativas, depende básicamente de una estructura operacional conformada por múltiples procesos y procedimientos técnicos y gerenciales, donde la información, es un elemento importante de constante cambio la cual marca e influye de manera radical la toma de decisiones que atribuyen al mejoramiento, a la creación de nuevas estrategias, normatividades, que según el caso deban ser de aplicabilidad.

Con respecto a la deserción estudiantil, bajo la perspectiva institucional, los análisis de CEDE (2012) consideran como desertor "aquel estudiante, que ha abandonado la institución educativa durante dos o más periodos consecutivos, sin haber reingresado hasta el momento en que se lleva a cabo la observación del sistema”. En este sentido, se derivan una serie de variables IPSA SCIENTIA - Vol. 5 No $1-2020$ 
individuales, académicas, institucionales, socioeconómicas y apoyos financieros determinantes de la deserción. Por su parte Sánchez Amaya (2007), sostiene que se entiende por abandono académico "al retiro forzado o voluntario que el estudiante hace de un programa académico o de la universidad”; mientras que la deserción se distingue como el abandono académico pero voluntario, que el estudiante hace de un programa o de la universidad.

Con respecto a los factores de deserción estudiantil, autores como Tapasco-Alzate et al. (2019), Zambrano-Verdesoto (2018), Barrero Rivera (2015), y Ruiz-Ramírez et al. (2014), coinciden en señalar que los mismos corresponden a la siguiente tipología:

- Individuales: relativos al ser, personalidad, habilidades, capacidades, intereses y motivaciones.

- Socioeconómicos; relativo a la influencia, trayectoria y rasgos del grupo familiar del estudiante.

- Institucionales-Académicos: marco de acción de la institución educativa, pensum de estudios, ambiente social, docentes, reglas de juego.

Por su parte, con base en lo establecido por el Ministerio de Educación Nacional a través del Sistema para la Prevención de la Deserción en las Instituciones de Educación Superior (SPADIES, 2014), se presentan los siguientes tipos de deserción estudiantil:

- Deserción por cohorte: Estudiantes que han sido marcados como desertores pertenecientes a una cohorte, luego de una cantidad de periodos determinada.

- Deserción por período: Cantidad de estudiantes que estando matriculados en $t$, se convirtieron en desertores en $\mathrm{t}+2$.

- Ausencia Intersemestral: Son todos aquellos estudiantes que no se matriculan en un semestre o aquellos que se dejan de matricular por un semestre.

\section{Materiales y Métodos}

Desde la óptica del estudio, se fija posición bajo el enfoque epistemológico fenomenológico, el cual busca la comprensión acerca de cómo funciona la conducta del individuo, mediante una interpretación de todas las situaciones cotidianas experimentadas, de tal forma que el investigador interprete, conozca las experiencias, vivencias, percepciones, significados, recuerdos, entre otros, del sujeto objeto de la indagación, dependiendo del tema o tópico tratado. (Leal, 2008).

Subsecuentemente, una vez enunciado el problema de investigación, y abordaje epistemológico como fenomenológico, se plantea el método o tradición del estudio, desde el paradigma científico cualitativo, que según Denzin \& Lincoln (2012), están de acuerdo con que lo cualitativo, debe permitir que "los datos les hablen", estar alerta y abiertos a cualquier cosa que emerja de ellos, forma característica de investigar, determinada por la intención sustantiva y el enfoque que la orienta. De manera que en la investigación cualitativa la fenomenología, es el método de la subjetividad en el que existe una visión de mundo enmarcada en el quehacer cotidiano con énfasis en lo individual y la experiencia subjetiva (Rodríguez, Gil \& García, 2006). 
En cuanto a la población objetivo, para este estudio en específico, corresponde a las instituciones de educación superior del sector privado de Barranquilla departamento del Atlántico. Bajo el criterio de empresas de carácter privado, por los cuales se pretende seleccionar a los dicentes claves, de acuerdo a las características de estas que son según su clasificación.

Los informantes clave, corresponden a los coordinadores académicos de estas instituciones. Para la clasificación de los informantes clave, se tomó en cuenta la conveniencia de trabajar con la Universidad Simón Bolívar en Barranquilla, y tres coordinadores académicos, los cuales aportan fundamentos sólidos ajustados a la realidad, por ello, el método fenomenológico proporciona una valiosa herramienta para establecer los criterios del entorno, así como, los datos que el informante está dispuesto a conceder a la investigación.

Asimismo, se propone la entrevista cualitativa, la cual tiene el doble propósito de construir la perspectiva de quien es estudiado y obtiene la información de la realidad que se desea conocer. Dentro de esta modalidad, se selecciona como instrumento el guión de entrevista. Para el análisis de los datos se empleó la categorización axial, que consiste en el proceso de relacionar las categorías a sus subcategorías, denominado axial, pues la codificación ocurre alrededor del eje de una categoría, enlaza a las categorías conforme a sus propiedades y dimensiones (Badilla, 2006).

\section{Resultados y Discusión}

\section{Categorización axial de los datos emitidos por los informantes clave}

Para esta categorización se agruparon las piezas discursivas, identificadas en el procedimiento anterior. Dicha categorización se presenta en los siguientes cuadros.

Cuadro 1. Categorización axial de los datos aportados por el participante 1

\begin{tabular}{|c|c|c|}
\hline Categorías & Subcategorías & Categorización axial \\
\hline \multirow{3}{*}{$\begin{array}{c}\text { Gerencia en la deserción } \\
\text { estudiantil }\end{array}$} & Habilidades gerenciales & $\begin{array}{c}\text { Deficientes competencias } \\
\text { en sus habilidades } \\
\text { gerenciales } \\
\text { Motivación intrínseca y } \\
\text { extrínseca } \\
\text { Solamente son operativos, } \\
\text { no proactivos }\end{array}$ \\
\hline & Factores de la deserción estudiantil & $\begin{array}{c}\text { Individuales } \\
\text { Socioeconómicos } \\
\text { Académicos } \\
\text { Institucionales } \\
\end{array}$ \\
\hline & Tipos de deserción estudiantil & $\begin{array}{c}\text { Por cohorte } \\
\text { Por periodo } \\
\text { Ausencia intersemestral }\end{array}$ \\
\hline
\end{tabular}

Fuente: elaboración propia

Con relación a los hallazgos mostrados en el cuadro 1, encontrados en las informaciones aportadas por el participante 1, después del proceso de reducción fenomenológica se infiere que la que la gerencia en la deserción estudiantil tienen algunas falencias en las habilidades IPSA SCIENTIA - Vol. 5 No $1-2020$ 
gerenciales, considerando que dentro de esta era del conocimiento, se debe gerenciar estratégicamente para dar respuesta al fenómeno del estudio. De acuerdo con este informante, se aplica la motivación pero, adolecen de capacidades y competencias organizativas para la toma de decisiones asertivamente. De igual manera, se mantienen los mismos axiomas de los factores y tipos de deserción en instituciones de educación del sector privado.

Cuadro 2. Categorización axial de los datos aportados por el participante 2

\begin{tabular}{|c|c|c|}
\hline Categorías & Subcategorias & Categorización axial \\
\hline & Habilidades gerenciales & $\begin{array}{c}\text { Deficientes competencias } \\
\text { en sus habilidades } \\
\text { gerenciales } \\
\text { Diagnostico } \\
\text { Motivación }\end{array}$ \\
\cline { 2 - 3 } $\begin{array}{c}\text { Gerencia en la deserción } \\
\text { estudiantil }\end{array}$ & Factores de la deserción estudiantil & $\begin{array}{c}\text { Socioeconómicos } \\
\text { Académicos } \\
\end{array}$ \\
\cline { 2 - 3 } & & Institucionales \\
\cline { 2 - 3 } & Tipos de deserción estudiantil & $\begin{array}{c}\text { Por cohorte } \\
\text { Por periodo }\end{array}$ \\
& & Ausencia intersemestral \\
\hline
\end{tabular}

Fuente: elaboración propia

Con respecto a la reducción fenomenológica mostrada en el cuadro 2 en relación a los informaciones aportadas en la categoría gerencia en deserción estudiantil, los hallazgos sugieren que no poseen habilidades gerenciales que le permita tomar decisiones asertivas para evitar este fenómeno por lo cual se hace necesario pensar estratégicamente, según las situaciones que se presentan. Asimismo, se mantienen las mismas doctrinas teóricas con respecto a los factores y tipos de deserción estudiantil en la institución objeto de investigación.

Cuadro 3. Categorización axial de los datos aportados por el participante 3

\begin{tabular}{|c|c|c|}
\hline Categorías & Subcategorías & Categorización axial \\
\hline \multirow{2}{*}{$\begin{array}{c}\text { Gerencia en la deserción } \\
\text { estudiantil }\end{array}$} & Habilidades gerenciales & $\begin{array}{c}\text { Insuficientes } \\
\text { competencias en sus } \\
\text { habilidades gerenciales } \\
\text { Motivación }\end{array}$ \\
\cline { 2 - 3 } & Factores de la deserción estudiantil & $\begin{array}{c}\text { Socioeconómicos } \\
\text { Académicos } \\
\text { Institucionales }\end{array}$ \\
\cline { 2 - 3 } & Tipos de deserción estudiantil & $\begin{array}{c}\text { Por cohorte } \\
\text { Por periodo }\end{array}$ \\
& Ausencia intersemestral \\
\hline
\end{tabular}

Fuente: elaboración propia

Los hallazgos según lo planteado por este participante (ver cuadro 3) derivaron del proceso de reducción fenomenología, sin embargo dicho informante no aporto información pertinente en referencia a la gerencia en la deserción estudiantil en instituciones de educación del sector privado, infiriendo de esta manera pocas competencias en las habilidades gerenciales, lo que significa que en el proceso de toma de decisiones gerencial no serían las más idóneas para contrarrestar este fenómeno. 
Sobre la base de estos resultados, en la mayoría de las subcategorías sujetas a estudio se generaron nuevas unidades de análisis que emergieron de la información suministrada por cada uno de los participantes, lo cual conlleva a volver a reestructuras los nuevos significados que surgieron para construir una teoría emergente. Los mapas conceptuales que se ilustran en las figuras 1 y 2 detallan el contraste resultante a partir de la información obtenida.

Figura 1. Subcategoría Habilidades Gerenciales

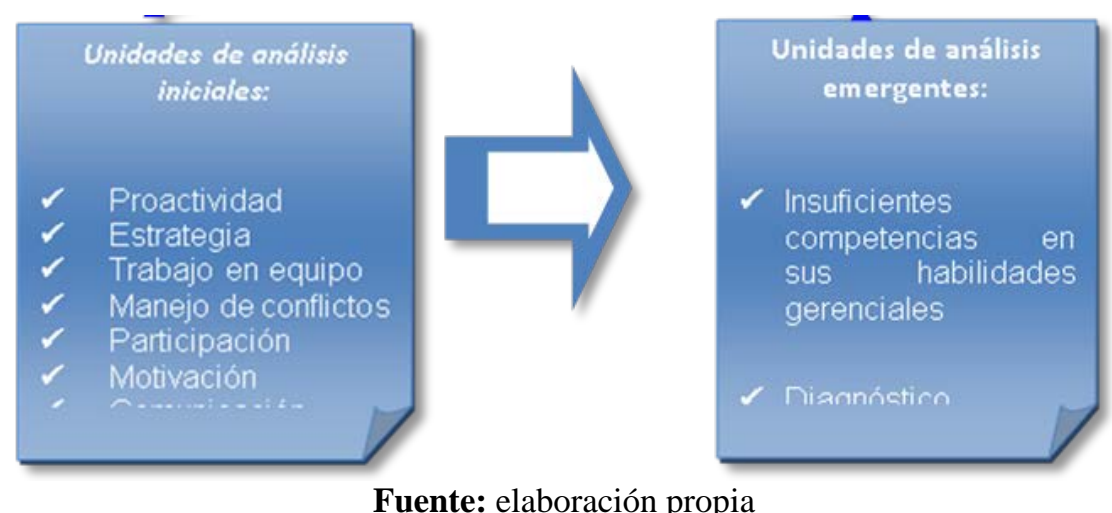

Fuente: elaboración propia

Figura 2. Subcategorías Factores en la deserción estudiantil y tipos de deserción

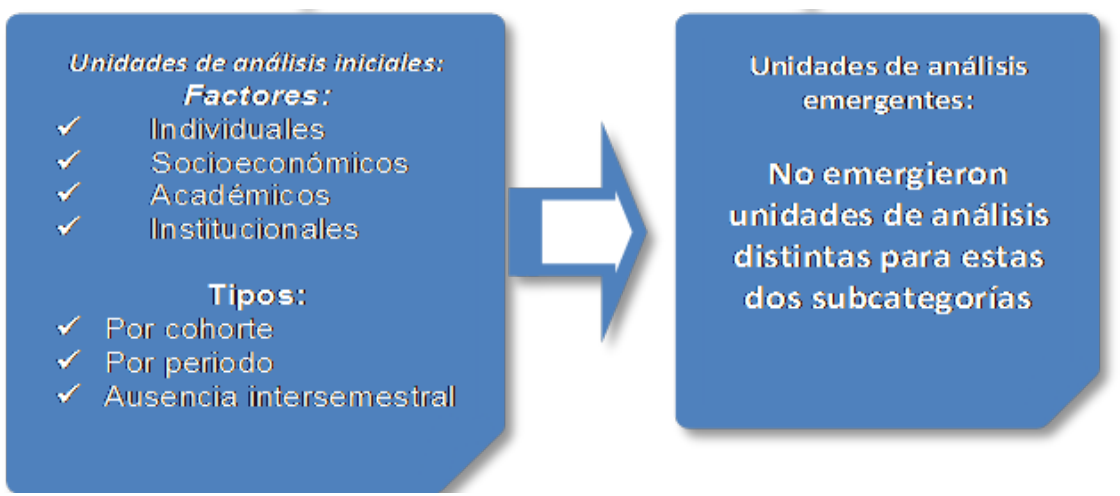

Fuente: elaboración propia

\section{Conclusiones}

En relación a explicar las habilidades gerenciales para la deserción estudiantil instituciones de educación superior del sector privado; se concluye que esta organización tiene pocos conocimientos de estas habilidades, lo cual es necesario que el recurso humano que coordina los programas de deserción estudiantil las posea, para poder tomar decisiones efectivas y asertivas en torno a este fenómeno.

Con respecto Categorizar los factores de la gerencia en la deserción estudiantil en instituciones de educación superior del sector privada, se concluye que se mantuvieron los mismos postulados teóricos, los cuales todas las instituciones de educación superior, los aplica, dado que son directrices del Ministerio de Educación Nacional de Colombia. 
En concordancia con el primer propósito: Explicar las habilidades gerenciales para la deserción estudiantil instituciones de educación superior del sector privado; en referencia con este aspecto se recomienda a las universidades, a fomentar sus habilidades gerenciales, desde una óptica estratégica capacitar al recurso humano, que permita contribuir a desarrollar elementos tácticos para tomar decisiones asertivas con relación a este tema tan fundamental en los entes universitarios.

Siguiendo con el segundo propósito: Categorizar los factores de la gerencia en la deserción estudiantil en instituciones de educación superior del sector privado; según lo aportado por los dicentes claves, desde sus experiencias en los programas de deserción, los estudiantes desertan por las mismas causas (económicas, individuales, académicas e institucionales).

\section{Referencias}

Agudelo, W. (2008). La educación en medios de comunicación. (Tesis doctoral). Universitat Rovira Virgili, Tarragona, España.

Badilla L (2006). Fundamentos del paradigma cualitativo en la investigación educativa. Revista de Ciencias del Ejercicio y la Salud, 4(1), 42-51.

Barrero Rivera, F. (2015). Investigación en deserción estudiantil universitaria: educación, cultura y significados. Revista Educación y Desarrollo Social, 9(2), 86-101.

CEDE, (2012). La deserción en la educación superior en Colombia durante la primera década del siglo XXI: ¿por qué ha aumentado tanto?. Bogotá, Colombia: Universidad de los Andes.

Clifton, R. A., Perry, R. P., Roberts, L. W., \& Peter, T. (2008). Gender, psychosocial dispositions, and the academic achievement of college students. Research in Higher Education, 49(8), 684-703.

Denzin, N. \& Lincoln,Y. (2012). Manual de investigación cualitativa. España: Editores Gedisa

Fong-Silva, W., Colpas-Castillo, F., \& Franco-Borré, D. (2018). Academic performance and its association with class attendance, intrinsic motivation and gender in engineering students. IPSA Scientia, Revista científica Multidisciplinaria, 3(1), 10-16. https://doi.org/10.25214/27114406.927

Franco-Gallego, J. (2019). Equidad en la educación superior colombiana desde el análisis de la graduación, 19982015. (Tesis doctoral). Universidad de La Salle, Bogotá, Colombia. Recuperado de https://ciencia.lasalle.edu.co/doct_educacion_sociedad/32

Kim Y.K., \& Sax L.J. (2017) The Impact of College Students' Interactions with Faculty: A Review of General and Conditional Effects. In: Paulsen M. (eds) Higher Education: Handbook of Theory and Research, 32. 85-139. Springer, Cham. https://doi.org/10.1007/978-3-319-48983-4_3

Leal, N. (2008). El método fenomenológico: principios, momentos y reducciones. Revista Electrónica de Investigación Científica, Humanística y Tecnológica, 1(5), 51-61.

Ley 1753. Por la cual se expide el Plan Nacional de Desarrollo 2014 - 2018. “Todos por un nuevo país”, del 9 de junio de 2015.2 Recuperado de https://colaboracion.dnp.gov.co/CDT/Normograma/Ley\%201753\%20de\%202015.pdf

Malagón, R. \& Reverón, C, (2001). La equidad social en la educación superior. Estadísticas e Indicadores de la Universidad Nacional de Colombia (2001)(5-6), 151-185.

IPSA SCIENTIA - Vol. 5 No $1-2020$ 
Rodríguez Gómez, G., Gil Flores, J. \& García Jiménez E. (2006). Metodología de la Investigación Cualitativa. Málaga: Ediciones ALJIBE.

Rodríguez, P., Mata, A., Jijón, W. \& García, M. (2017). Influencia de la educación en el desarrollo económico: Caso Distrito Metropolitano de Quito. Revista Publicando, 4(12 (1)), 512-522.

Ruiz-Ramírez, R.; García-Cué, J.; Pérez-Olvera, M. (2014). Causas y consecuencias de la deserción escolar en el bachillerato: caso Universidad Autónoma de Sinaloa. Ra Ximhai, 10(5), 51-74.

Sánchez Amaya, G. (2007). Factores de deserción estudiantil en la Universidad Surcolombiana: sede Neiva 20022005. Neiva, Colombia:Universidad Surcolombiana..

Sistema para la Prevención de la Deserción en las Instituciones de Educación Superior -SPADIES (2014). Manual de conceptos sistemas de información SPADIES. Ministerio de Educación Nacional Colombia. Recuperado de http://www.mineducacion.gov.co/sistemasdeinformacion/1735/articles268156_archivo_pdf_Manual_sincronizacion.pdf

Tapasco Alzate, O. A., Ruiz Ortega, F. J., Osorio García, D., \& Ramírez Ramírez, D. (2019). Deserción estudiantil: incidencia de factores institucionales relacionados con los procesos de admisión. Educación y Educadores, 22(1), 81-100.

Zambrano Verdesoto, G., Rodríguez Mora, K., \& Guevara Torres, L. (2018). Análisis de la deserción estudiantil en las universidades del Ecuador y América Latina. Revista Pertinencia Académica. (8), 01-28. Recuperado de http://revista-academica.utb.edu.ec/index.php/pertacade/article/view/127 